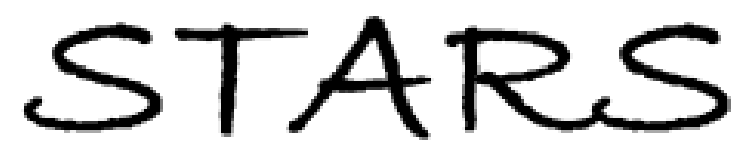

University of Central Florida

STARS

$1-1-2006$

\title{
Enhanced photonic band edge laser emission in a cholesteric liquid crystal resonator
}

\author{
Ying Zhou \\ University of Central Florida \\ Yuhua Huang \\ University of Central Florida \\ Zhibing Ge \\ University of Central Florida \\ Liang-Pin Chen \\ University of Central Florida \\ Qi Hong \\ University of Central Florida \\ Find similar works at: https://stars.library.ucf.edu/facultybib2000 \\ University of Central Florida Libraries http://library.ucf.edu \\ See next page for additional authors \\ This Article is brought to you for free and open access by the Faculty Bibliography at STARS. It has been accepted for \\ inclusion in Faculty Bibliography 2000s by an authorized administrator of STARS. For more information, please \\ contactSTARS@ucf.edu.
}

\section{Recommended Citation}

Zhou, Ying; Huang, Yuhua; Ge, Zhibing; Chen, Liang-Pin; Hong, Qi; Wu, Thomas X.; and Wu, Shin-Tson, "Enhanced photonic band edge laser emission in a cholesteric liquid crystal resonator" (2006). Faculty Bibliography 2000s. 6775.

https://stars.library.ucf.edu/facultybib2000/6775

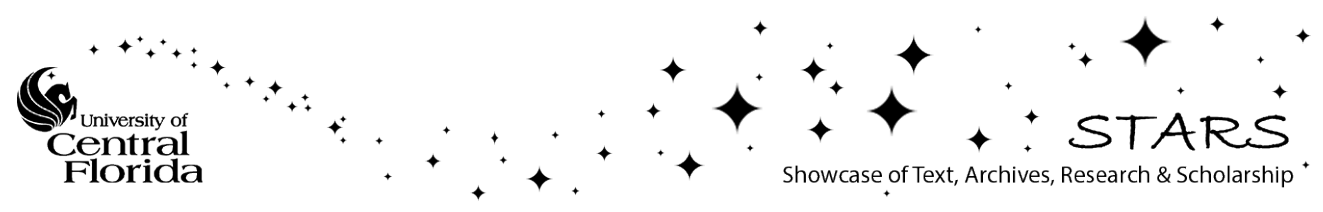


Authors

Ying Zhou, Yuhua Huang, Zhibing Ge, Liang-Pin Chen, Qi Hong, Thomas X. Wu, and Shin-Tson Wu 


\title{
Enhanced photonic band edge laser emission in a cholesteric liquid crystal resonator
}

\author{
Ying Zhou, Yuhua Huang, Zhibing Ge, Liang-Pin Chen, Qi Hong, Thomas X. Wu, and Shin-Tson Wu \\ College of Optics and Photonics, University of Central Florida, Orlando, Florida 32816, USA
}

(Received 31 July 2006; revised manuscript received 25 September 2006; published 26 December 2006)

\begin{abstract}
A highly efficient photonic band edge dye-doped cholesteric liquid crystal (CLC) laser is demonstrated. By sandwiching an active CLC cell within a resonator consisting of two passive CLC reflectors, the lasing efficiency is dramatically enhanced. Theoretical analysis using the improved $4 \times 4$ transfer matrix and scattering matrix shows that the band edge laser mode can be supported by the external CLC resonator and its optimal output can be achieved by a relatively thin active CLC layer and thin passive CLC reflectors. Theoretical analysis agrees well with the experimental results.
\end{abstract}

DOI: 10.1103/PhysRevE.74.061705

PACS number(s): 61.30.- $-\mathrm{v}, 42.55 .-\mathrm{f}$

\section{INTRODUCTION}

Photonic crystals, showing an ordered structure with a periodic modulation of refractive index, have attracted tremendous interests from both scientific and technological viewpoints. In these materials, propagation of photons is forbidden within a certain frequency range, known as photonic band gap (PBG). Cholesteric liquid crystal (CLC) is regarded as anisotropic one-dimensional (1D) photonic crystal since the liquid crystal (LC) directors are self-organized into a helical structure [1]. Bragg reflection is established in cholesteric planar structure as the result of alternating ordinary and extraordinary refractive index. Selective reflection band is thus induced for the circularly polarized light in the same sense as the cholesteric helix. Such a reflection band can be accurately determined by simple relationships as $\lambda_{0}=\langle n\rangle p$ and $\Delta \lambda=\Delta n p$, where $\lambda_{0}$ and $\Delta \lambda$ represent the central wavelength and bandwidth of the reflection band, $\langle n\rangle$ and $\Delta n$ are the average refractive index and birefringence of the CLC, respectively; and $p$ is the intrinsic helical pitch length [2]. Based on these properties, CLC has been widely investigated for display and photonic applications, e.g., bistable reflective displays, circularly polarized reflectors, and tunable lasers [3-6]. For photonic applications, the strong localization of photons at photonic band edge opens a way for generating laser light where CLC provides a distributed feedback. Extensive work has been conducted on dye-doped CLC photonic band edge lasers $[7,8]$. On the other hand, low-threshold laser emission has also been observed by introducing a defect layer, either isotropic [9] or anisotropic [10,11], into the CLC based resonator. Lowering the lasing threshold can also be achieved by putting a dye-doped cholesteric liquid crystal between two polymer films with one reflecting the lasing wavelength and the other reflecting the pumping wavelength [12].

Without a special fabrication process, the defect-free cholesteric planar structure we have obtained so far is limited to $\sim 10 \mu \mathrm{m}$ cell gap due to the insufficient surface anchoring energy. Because of this short cavity length, the lasing efficiency is quite limited and the laser beam is highly divergent. To overcome these shortcomings, adding a passive reflector is proven to be helpful [13].

In this paper, we use an external resonator which consists of two passive CLC reflectors to enhance the laser emission from a single dye-doped CLC cell. Transmission spectrum of three cholesteric polymeric layers with different pitches was previously calculated by Ozaki et al. [14], but no further work in laser generation and output performance was reported so far. Hence, we not only demonstrate an enhanced emission from the active CLC cell sandwiched within a CLC resonator but also theoretically present the responsible physical mechanisms of the laser mode and light amplification. Key parameters in such a CLC laser system as the thickness of the active CLC middle layer and the amplification index constant are investigated to provide guidelines for the system optimization. In addition, the effect of inserting glass substrates on the laser performance is analyzed in detail.

\section{EXPERIMENT}

The active CLC sample was prepared by using a Merck nematic LC host BL006 $\left(\Delta n=0.286, \quad n_{e}=1.826 @ \lambda\right.$ $=589 \mathrm{~nm}$ and $T=20^{\circ} \mathrm{C}$ ) and $27.3 \%$ of right-handed chiral agent MLC6248 (Helical twisting power $=11.3 \mu \mathrm{m}^{-1}$, from Merck). The mixture was then doped with $1.5 \mathrm{wt} . \%$ laser dye DCM (4-(Dicyanomethylene)-2-methyl-6-(4dimethylaminostyryl)-4H-pyran, from Exciton) as the emissive medium. The passive CLC reflectors were prepared using BL006 and $25.3 \%$ of MLC6248. The mixture was filled into a $5 \mu \mathrm{m}$ homogenous cell by capillary action under isotropic state. The inner surfaces of the cells were first coated with ITO (Indium-Tin-Oxide) layers and then polyimide alignment layers, which were rubbed in anti-parallel directions to produce a small $\left(\sim 3^{\circ}\right)$ pretilt angle. The filled CLC cells were slowly cooled down to room temperature using a temperature controller in order to form a defect-free helical structure. In this work, the $5 \mu \mathrm{m}$ active CLC cell was sandwiched between two $5 \mu \mathrm{m}$ CLC reflectors in optical contact to form a three-cell CLC laser assembly, as Fig. 1 shows. The cells were stacked together tightly using a demountable holder which has a screw in each of the four corners for controlling the cell uniformity. No special process for monitoring the parallelism was performed during cell assembly. In our experiment, it is not difficult to find the emission point with a dramatically enhanced output; the whole assembly is not as sensitive to parallelism as the structure which has an anisotropic emissive layer in the middle (e.g., the dye-doped nematic liquid crystal). This is because the middle CLC layer 


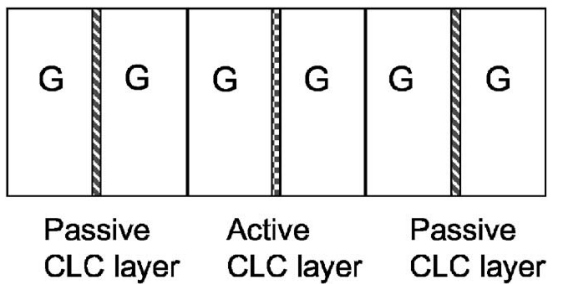

FIG. 1. The structure of a three-CLC cell laser assembly. $G$ represents the glass substrate. In experiment $d_{\text {active CLC }}$ $=d_{\text {passive CLC }}=5 \mu \mathrm{m} ; d_{\text {glass substrate }}=1.1 \mathrm{~mm}$.

lases itself and the feedback comes from both the middle CLC layer and the passive reflectors.

Figure 2 shows the experimental setup. A secondharmonic $Q$-switched Nd:YAG pulsed laser (Minilite II, from Continuum) operated at $\lambda=532 \mathrm{~nm}, 4 \mathrm{~ns}$ pulse width, and $1 \mathrm{~Hz}$ repetition rate was used to pump the CLC cell at normal incidence. The pump beam was separated into two paths: one was monitored by an energy meter (Laserstar, from Ophir) and the other was used as the pump beam. In order to reduce the Bragg reflection from the tested CLC laser whose reflection band may cover the pump wavelength, the linearly polarized pump beam was converted into a lefthanded circularly polarized (LCP) beam before it was focused onto the CLC cells. The pump spot on the samples was estimated to be $\sim 160 \mu \mathrm{m}$ in diameter. Laser emission in forward direction was then collected by a focusing lens into a fiber-based spectrometer (HR2000, from Ocean Optics, resolution $\sim 1 \mathrm{~nm}$ ). Such a configuration would reduce the surface reflection and, moreover, the laser emission after multiple reflections inside the CLC resonator can still be aligned into one spot with a relatively small divergence. On the other hand, the oblique pump results in multiple reflections of the pumping beam $(532 \mathrm{~nm})$ inside the CLC resonator due to the glass surfaces. Then the gain medium will be pumped by each reflected beam so that multiple laser beams are generated in a row with gradually decreased power. This phenomenon is indeed observed in our experiment.

Figure 3 depicts the normalized transmittance of the active CLC cell (thin solid line) and the passive CLC reflector (dotted line), from which the reflection band can be clearly characterized. With DCM doped, the short wavelength band edge of the active CLC cell is completely obscured by the absorption of the laser dye. The reflection band of the $5 \mu \mathrm{m}$ CLC reflector is chosen to cover the lasing wavelength and its measured reflectivity at $\lambda=600 \mathrm{~nm}$ is $\sim 97 \%$. As an example, the lasing spectrum at $33.4 \mu \mathrm{J} /$ pulse pump energy is

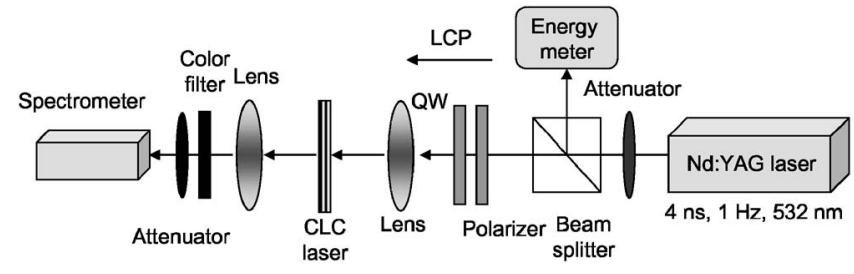

FIG. 2. Experimental setup of a CLC laser assembly at normal incidence; QW: quarter-wave plate, and LCP: left-handed circularly polarized light.

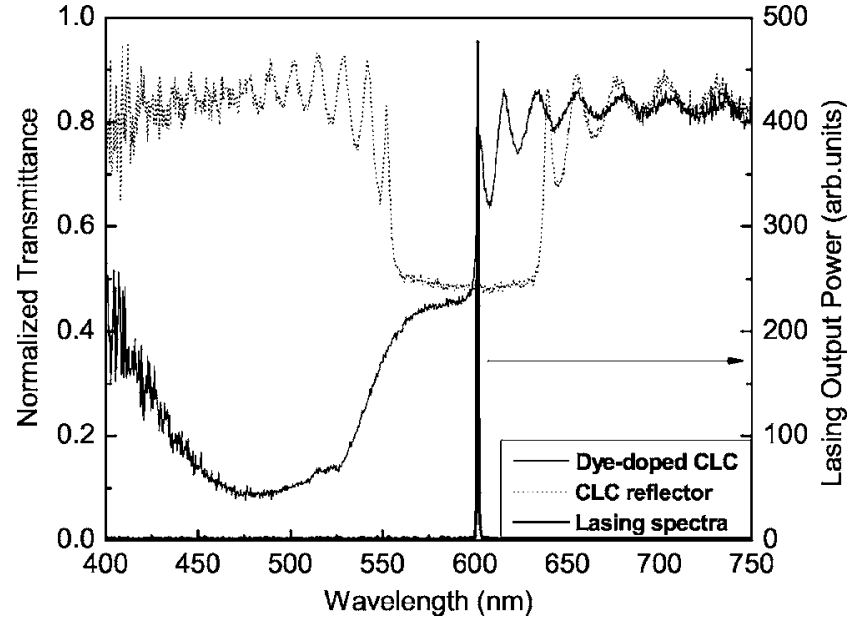

FIG. 3. Measured normalized transmittance of the dye-doped CLC cell (thin solid line), the measured normalized transmittance of passive CLC reflector (thin dotted line), and the emission spectra of CLC laser assembly pumped at $33.4 \mu \mathrm{J} /$ pulse (thick solid line).

also plotted (thick solid line) in the same figure to allow an easy justification for lasing action at the photonic band edge.

Laser output power at different pump energy for the $5 \mu \mathrm{m}$ CLC laser within a CLC resonator was measured. Results are plotted in Fig. 4 as marked by triangles. To compare the enhancement factor, the laser output power of a single $5 \mu \mathrm{m}$ CLC cell (squares), as well as the $5 \mu \mathrm{m}$ CLC laser with one CLC reflector (circles) was measured. From Fig. 4, the single $5 \mu \mathrm{m}$ dye-doped CLC cell generates a weak laser emission because of its thin CLC emissive layer and short feedback length. With the help of a CLC reflector incorporated in good contact at one end, the laser emission from one direction is reflected back into the cavity. Because the circular polarization state of the reflected beam is preserved by the "polarization-conserved CLC reflector" and is further amplified by the cavity, the output emission is dramatically en-

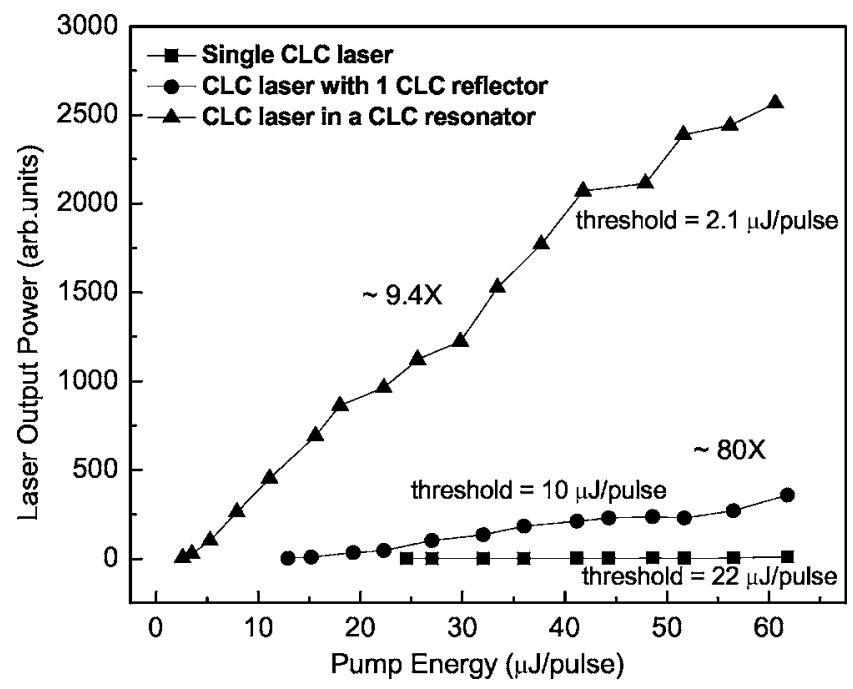

FIG. 4. Pump energy dependent laser output power of a single $5 \mu \mathrm{m}$ active CLC cell (squares), a $5 \mu \mathrm{m}$ active CLC cell with a $5 \mu \mathrm{m}$ passive reflector enhanced (circles), and a $5 \mu \mathrm{m}$ active CLC cell within a CLC circularly polarized resonator (triangles). 

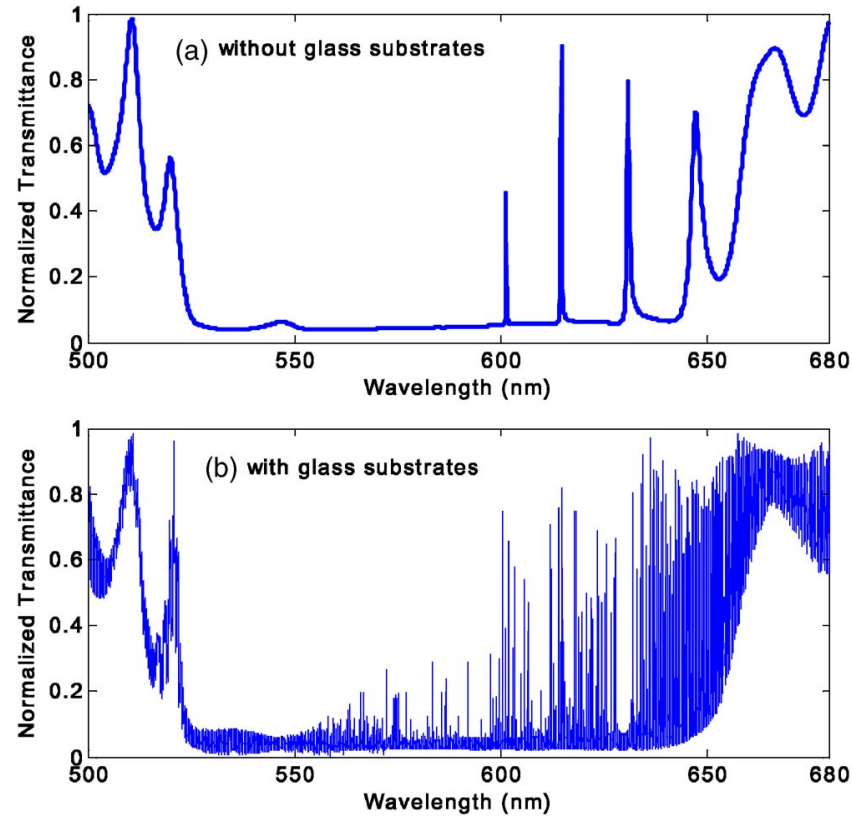

FIG. 5. (Color online) Simulated normalized transmittance of the passive 3-CLC assembly: (a) with no glass substrates present for each cell, immersed in air; (b) with glass substrates for each cell, immersed in air.

hanced [13]. Under this circumstance, the output is still right-handed circularly polarized, the same as its original polarization state. The observed laser emission is $\sim 80 \mathrm{X}$ stronger as compared to the emission from the single active CLC alone. Furthermore, we found that when the active CLC cell is confined between two CLC reflectors, the output emission is further enhanced by $\sim 9.4 \mathrm{X}$ as detected from one end of the laser cavity. Because of the structure symmetry, the laser output has equal power coming from both ends. The lasing wavelength is located at $\lambda=602 \mathrm{~nm}$, exactly at the long wavelength band edge of the middle CLC lasing cell and it is elliptically polarized. Meanwhile, as the right-handed cholesteric reflector is incorporated to the single CLC laser from one side to two sides, the lasing threshold decreases from $22 \mu \mathrm{J} /$ pulse to $10 \mu \mathrm{J} /$ pulse, and finally down to $2.1 \mu \mathrm{J} /$ pulse. The reduction is about one order of magnitude.

\section{THEORETICAL SIMULATIONS}

\section{A. Simulation results of the passive CLC assembly}

To understand how the external resonator enhances the band edge lasing of the middle CLC cell, we calculate the transmittance and the coherent amplification of light for different polarizations using improved $4 \times 4$ transfer matrix and scattering matrix. In this method, the $4 \times 4$ matrix for each slice is diagonalized and thus the equations become very concise. Detailed derivations can be found in Refs. $[15,16]$. The employed method rigorously takes into account the boundary conditions and multiple interface reflections of the LC medium.

Figure 5 is a plot of normalized transmittance of a passive three-CLC assembly without doping DCM laser dye. In or- der to see the characteristic spectral lines introduced by Fabry-Perot (FP) effect from the resonator, we first calculate the transmittance of three CLC layers directly attached to each other, assuming that glass substrates are absent. Results are shown in Fig. 5(a). Afterwards, the transmittance of three real CLC cells (with glass substrates) is calculated as Fig. 5(b) shows. The thickness of each glass substrate is $1.1 \mathrm{~mm}$. Some basic parameters used for simulation are listed as follows: refractive index of air $n_{\text {air }}=1$, and LC $n_{o}=1.50, n_{e}$ $=1.70$; the active CLC middle layer is right handed with pitch length $p_{1}=0.35 \mu \mathrm{m}$ and cell gap $d_{1}=5 \mu \mathrm{m}$; the two CLC reflectors are also right handed with pitch length $p_{2}$ $=0.375 \mu \mathrm{m}$ and cell gap $d_{2}=2.25 \mu \mathrm{m}$ in order to reach $\sim 96.5 \%$ reflectivity. The incident light is right-handed circularly polarized (RCP). Doping 25\%-27\% of the chiral material into nematic LC host causes a decrease in birefringence of the whole CLC mixture to $\sim 0.2$. Although the LC refractive indices and the birefringence used for simulations are somewhat different from those of BL006, the simulation result of a single CLC layer does give a reflection band from 525 to $600 \mathrm{~nm}$ for the middle CLC layer and $560-640 \mathrm{~nm}$ for the passive CLC reflectors. These simulated results agree with experimental data reasonably well.

From Fig. 5(a), when the right-handed CLC reflectors are present at both ends, the resonator produces several transmission peaks in the $600-640 \mathrm{~nm}$ range. In other words, the resonance modes only exist within the reflection band of the passive reflectors $(\sim 560-640 \mathrm{~nm})$ which does not overlap with that of the middle CLC $(\sim 525-600 \mathrm{~nm})$. The mode spacing corresponds to a Fabry-Perot whose cavity length is equal to the total thickness of the three CLC layers ( $d$ $=9.5 \mu \mathrm{m}, n=1.6)$. However, within the overlapping reflection band of the active CLC middle layer and the passive CLC reflectors (ranged 560-600 $\mathrm{nm}$ ), the transmission peaks are completely suppressed except the one at the band edge $(600 \mathrm{~nm})$. More simulation results (not shown here) indicate that there always appears a transmission peak near the band edge even if the band edge does not coincide with the FP transmission peak introduced by the CLC resonator. This means the photonic band edge mode of the active CLC can be well preserved and, more importantly, be further strengthened by the external resonator.

As Fig. 5(b) shows, with glass substrates present the increased FP length brings in a lot of oscillations, the extra possible resonance modes. They function as high frequency modulation superimposed onto the original characteristic spectral lines. Yet the envelope of these oscillations can still be distinguished to match the characteristic spectral lines assuming that the spectrum step for calculation is small enough.

These oscillations are not randomly distributed; instead they ensue from the several embedded FP cavities built up with CLC layers and the glass substrates. Figure 6 shows a detailed outlook of these oscillation modes within $614.2-614.8 \mathrm{~nm}$ range as an example, where one of the characteristic spectral lines of the three-CLC layer assembly locates [see from Fig. 5(a)].

For a typical FP, the free spectral range $\Delta \lambda_{l}$ can be calculated according to the equation $\Delta \lambda_{l}=\frac{\lambda_{l}^{2}}{2 n d}[17]$, where $\lambda_{l}$ is the 


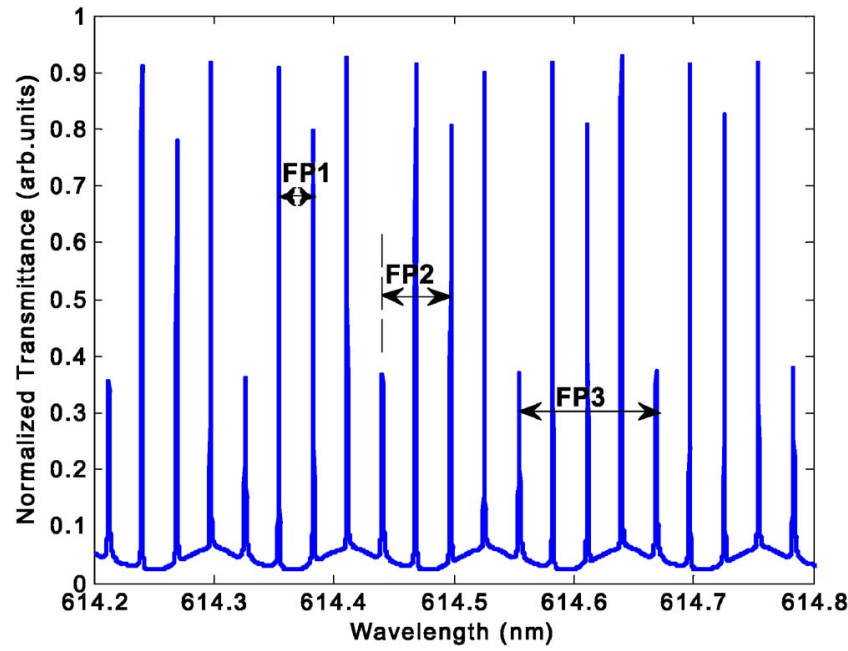

FIG. 6. (Color online) Detailed view of simulated normalized transmittance of the passive three-CLC assembly with glass substrates $(n=1.5)$ in detail.

wavelength of interest, $n$ is the refractive index, and $d$ is the gap of the FP cavity. From Fig. 6, we found different free spectral ranges superimposed on each other. There are basically three FP cavities contributing to these oscillation peaks: (1) FP1, formed by the two passive CLC layers ( $d=$ $\sim 4.4 \mathrm{~mm}$ ), corresponding to $\Delta \lambda_{l}=0.028 \mathrm{~nm}$. This is the most dominant factor in the spectrum. (2) FP2, formed by one passive CLC layer and the active CLC layer $(d=$ $\sim 2.2 \mathrm{~mm}$ ), corresponding to $\Delta \lambda_{l}=0.056 \mathrm{~nm}$. The wavelength $\lambda_{l}(=614 \mathrm{~nm})$ we investigate here is actually beyond the reflection band of the active CLC in the middle $(525-600 \mathrm{~nm})$, whereas due to Bragg reflection the side oscillation near its band edge causes $\sim 3.3 \%$ reflectivity for $\mathrm{RCP}$ at $614 \mathrm{~nm}$. This is why the active and the passive CLC layers still form a FP even the wavelength is outside the reflection band of one of the two CLC layers. As a result, it superimposes the lower frequency oscillations onto the transmission spectrum from FP1. (3) FP3, formed between the air-glass interface and its neighboring passive CLC layer $(d$ $=\sim 1.1 \mathrm{~mm}$ ). This corresponds to $\Delta \lambda_{l}=0.112 \mathrm{~nm}$. The total effect of three embedded FPs is that the transmission peaks with a higher frequency oscillation as from FP1 are modulated by the lower frequency oscillations as from FP2 and FP3.

In theory, the reflectivity of a $2.25 \mu \mathrm{m}$ CLC reflector should already reach $96.5 \%$ for the RCP incidence at its reflection band center $(\lambda \sim 600 \mathrm{~nm})$ although the Bragg reflection is not completely established. In reality, our CLC reflector is $5 \mu \mathrm{m}$ thick but its reflectivity at $600 \mathrm{~nm}$ was measured to be $\sim 97 \%$ because of the imperfect helical distribution of LC directors. Besides, the possible air gap between the CLC cells in experiment may further decrease the reflectivity. In our simulation the effect of ITO layer was not taken into consideration because a typical ITO layer with index $\sim 1.7$ and thickness $\sim 100 \mathrm{~nm}$ will only decrease the reflectivity of our CLC reflector (at $600 \mathrm{~nm}$ ) by $\sim 0.5 \%$, which will not significantly affect the results. Simulation also reveals that to further increase the cell gap of the CLC reflector to $8 \mu \mathrm{m}$ does not generate any stronger laser emission because the optimal output for FP not only requires a high reflectivity but also an indispensably small transmittance so as not to block all the output. Experimental measurement of transmittance does not exhibit these narrow transmission peaks clearly other than some undulations because the resolution of the spectrometer $(\sim 1 \mathrm{~nm})$ is much larger than the band width of the transmission peaks $(\sim 0.2 \mathrm{~nm}$ at $\lambda=601 \mathrm{~nm})$.

\section{B. Simulation results of the active CLC assembly}

To simulate the light amplification behavior inside the dye-doped CLC layer, the refractive index of the active CLC is modified to calculate the normalized transmittance of three CLC cells assembly. According to the plane wave definition $\vec{E}=\vec{E}_{0} \exp [-i(\omega t-k z)]$ (with $\vec{E}_{0}, \omega, k$, and $z$ representing the amplitude, frequency, propagation constant, and distance, respectively), a negative imaginary part is added to the refractive index of each sliced CLC layer as $n_{o}=n_{o}-n^{\prime \prime} i$ and $n_{e}$ $=n_{e}-n^{\prime \prime} i\left(n^{\prime \prime}>0\right)$. In calculations, this imaginary part functions equivalently as a gain parameter of the gain medium inside the LC. Thus, the onset of laser oscillation is indicated by the divergence of the transmittance and the light amplification is represented by a normalized transmittance larger than unity. The complex relationship between the amplification index constant $n^{\prime \prime}$ and what follows the real fluorescent material parameters, together with the output intensity are out of our current research scope. For simplicity, we can choose $n^{\prime \prime}$ to be a frequency independent constant. Simulation under such an assumption was validated in some other literatures [18-20]. The amplification index constant $n^{\prime \prime}$ should not be too large to ensure the convergence of the program. In our simulation, we set $n^{\prime \prime}=0.0001$.

Without glass substrates present, simulation results with RCP and LCP incidence are shown in Figs. 7(a) and 7(b), respectively, where the light amplification at the band edge wavelength $(\sim 601 \mathrm{~nm})$ can be observed for both RCP and LCP. This is attributed to the high reflectivity of RCP from the right-handed CLC and the low reflectivity of LCP from the interface reflection of the air and the CLC layer. As a consequence, the light near the band edge can be effectively amplified by the external resonator for both orthogonal polarizations. The final output beam is elliptically polarized owing to the superposition of both amplified RCP and LCP beams.

With glass substrates present, the light also gets amplified at the band edge $(\sim 601 \mathrm{~nm})$ for both RCP and LCP incidences as shown in Figs. 8(a) and 8(b), which resembles the phenomena in the case without glass substrates. The peak power at the amplified mode reaches almost the same value as that from Fig. 7. In addition, a preliminary measurement on the beam divergence half angle has shown a remarkable decrease from $\sim 4.5^{\circ}$ (single CLC cell) to $\sim 0.5^{\circ}$ (three-CLC cells assembly). Together with Fig. 5(b), we note that the effects caused by glass substrates are summarized as follows: (1) a lot of oscillations are superimposed onto the characteristic spectral lines whereas the main features still remain. Therefore, introducing the substrates will not obviously pull the amplified mode away; (2) ideally, introducing the glass 

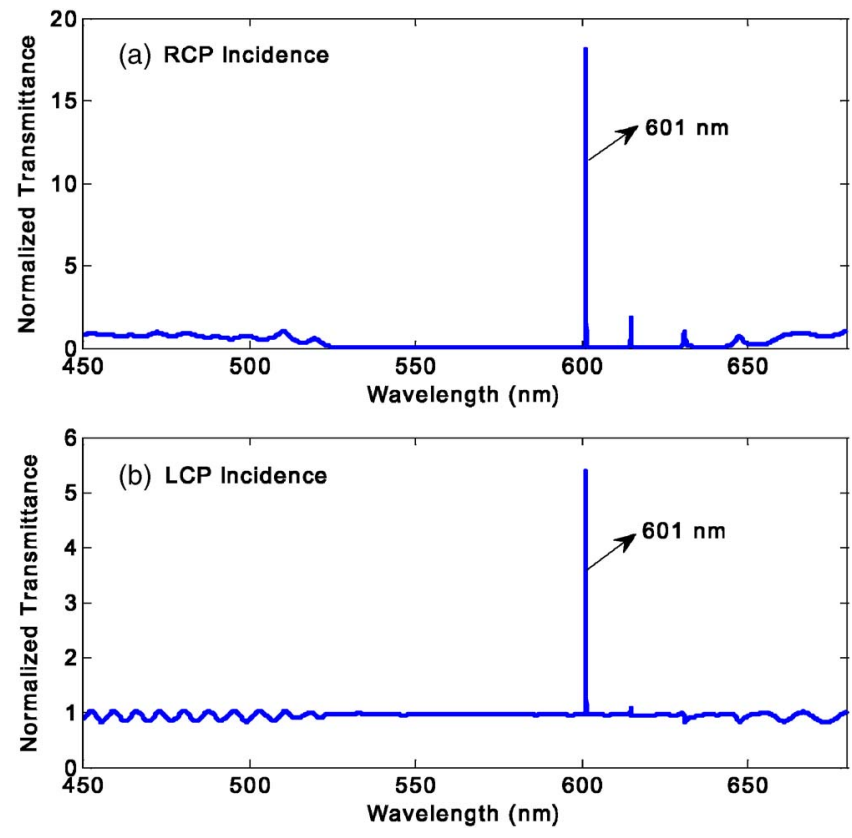

FIG. 7. (Color online) Simulated normalized transmittance with amplifying medium in the middle CLC cell without glass substrates: (a) RCP as incidence; (b) LCP as incidence. Peak wavelength is at $601 \mathrm{~nm}$.

substrates into the CLC assembly will not noticeably decrease the peak power of the dominant amplified mode because the refractive index of glass is close to the average refractive index of CLC. However, the air gaps between the CLC cells in experiment may slightly deteriorate the lasing output power; and (3) the increased cavity length to $\mathrm{mm}$ scale dramatically decreases the beam divergence. Compared to the CLC cells assembly, such a structure can be realized using three stacked cholesteric polymer films (one active film between two passive films) as well. The major advantage of such a configuration is that the glass substrates can be completely removed and the device can be very thin [21]. However, to fabricate such cholesteric polymer films a UV curing process is commonly practiced so that the dye bleaching problem during UV exposure is a concern. Moreover, the employed LC polymer usually has a smaller birefringence (e.g., $\Delta n \sim 0.1$ for the Merck chiral monomers RMM154 and RMM153) than BL006 $(\Delta n \sim 0.286)$. As a result, the overall lasing efficiency would be lower and beam divergence larger than the corresponding three CLC cells assembly. This is indeed observed in our experiment.

In order to find out the optimal parameters for the CLC resonator and the active CLC cell, we investigate the amplification performance as a function of the amplification index constant $n^{\prime \prime}$ and the thickness of the active CLC cell $d_{1}$ using RCP as the incidence. Figures 9(a)-9(d) give the results of light amplification as $n^{\prime \prime}$ gradually increases. As shown in Figs. 9(a) and 9(b), at smaller $n^{\prime \prime}$ the incident RCP light gets amplified at the band edge wavelength of the active CLC cell (the mode at $600 \mathrm{~nm}$ ). As $n^{\prime \prime}$ increases, the amplification gets stronger. However, further increasing $n^{\prime \prime}$ does not give rise to a stronger emission; on the contrary, it weakens the amplification performance accompanied by a mode shift. As Figs.
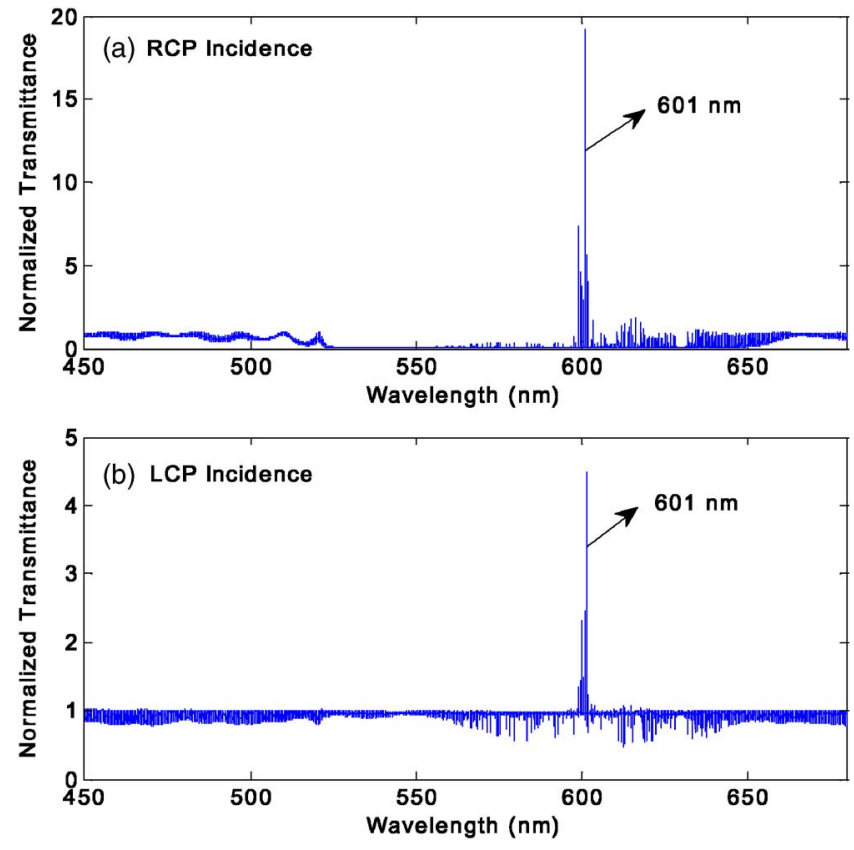

FIG. 8. (Color online) Simulated normalized transmittance with amplifying medium in the middle CLC cell with glass substrates: (a) RCP as incidence; (b) LCP as incidence. Peak wavelength is at $601 \mathrm{~nm}$.

9(c) and 9(d) show, the mode closer to the band edge of the CLC reflector (the mode at $640 \mathrm{~nm}$ ) becomes more predominant as $n^{\prime \prime}$ increases. This implies that within the reflection band of the resonator the PBE mode (at $600 \mathrm{~nm}$ ) of the active CLC is easier to be amplified than the band edge mode (at $640 \mathrm{~nm}$ ) of the passive reflector. This trend is in accordance with the amplification behavior of the resonance modes in a typical FP, assuming a wavelength independent gain. The calculation of a simple FP with a complex refractive index for the medium between two end mirrors shows that the mode near the reflection band center is amplified at a smaller $n^{\prime \prime}$ value while the one near the band edge is amplified at a larger $n^{\prime \prime}$ value. However, in practice the PBE mode $(600 \mathrm{~nm})$ appears first and this mode will suppress the other possible laser modes. Therefore, in experiment only the mode at $600 \mathrm{~nm}$ can be readily observed other than the modes from FP effect of the resonator. On the other hand, the fluorescence peak of the emissive medium DCM is around $590-600 \mathrm{~nm}$. For the laser modes beyond this range, the fluorescent intensity drops quickly, which also contributes to the dominant band edge mode.

By varying the thickness of the active CLC cell in the middle, the tendency is similar to the behavior exhibited in Figs. 9(a)-9(d). Here $n^{\prime \prime}$ is again fixed at 0.0001. From Figs. 10(a) and 10(b), as the thickness of the active CLC layer is increased from 5 to $7 \mu \mathrm{m}$, the RCP incident light is amplified at the band edge of the active CLC cell and the lasing strength is boosted by $\sim 6 \mathrm{X}$. While beyond a certain value, the dominant amplified mode shifts towards the band edge of the CLC reflector and the peak power decreases. This critical thickness, above which the mode shift starts to occur, is of course relying on the setting value of $n^{\prime \prime}$. The $n^{\prime \prime}$ in our cur- 

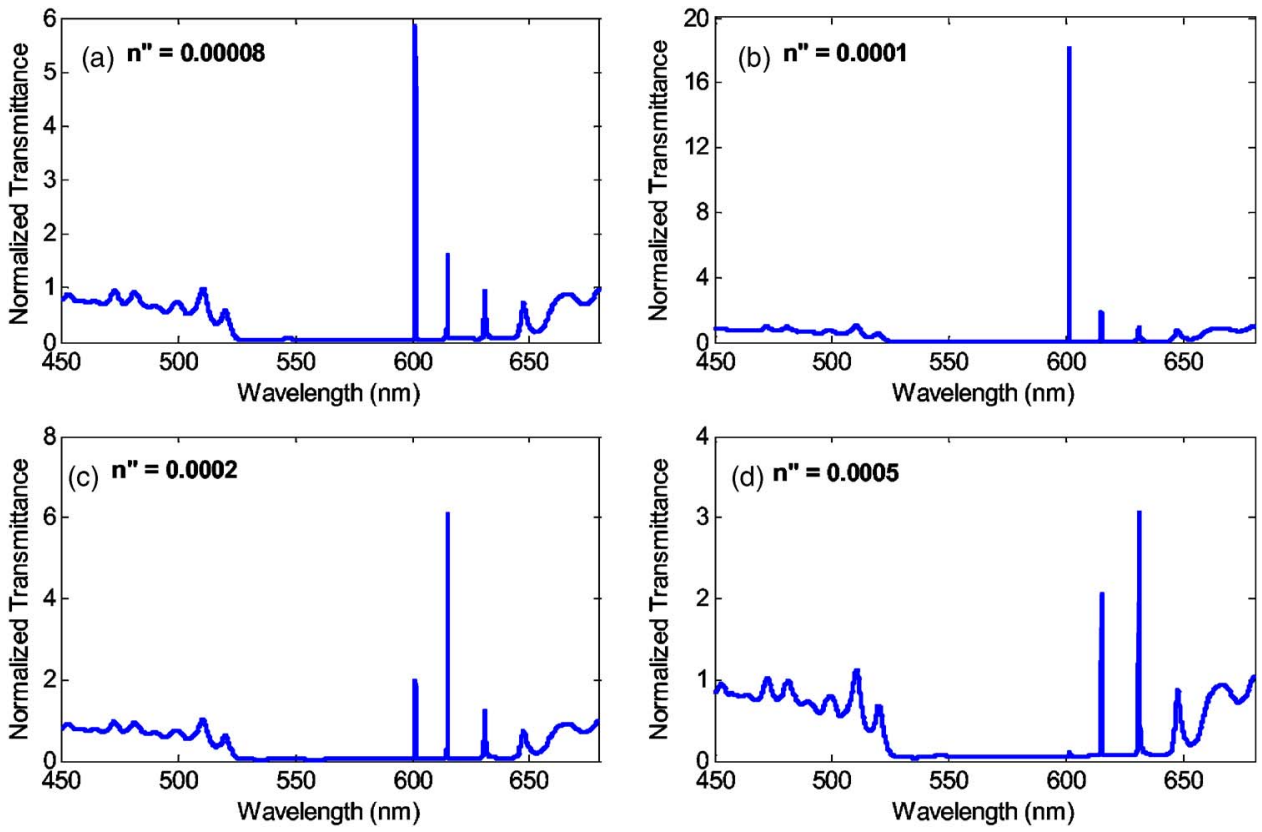

FIG. 9. (Color online) Amplification index constant $n \prime \prime$ dependent light amplification performance: (a) $n^{\prime \prime}=0.00008$; (b) $n^{\prime \prime}$ $=0.0001 ;$ (c) $n^{\prime \prime}=0.0002 ;$ (d) $n^{\prime \prime}$ $=0.0005$. The incidence is RCP. rent calculations is not directly correlated to the dye's absorption, fluorescence, and the population inversion, nevertheless the tendency found here is again confirmed by the fact that we did not experimentally obtain any stronger emission from the $8 \mu \mathrm{m}$ or thicker active CLC laser in the resonator. Increasing the thickness of the active CLC cell is equivalent to increasing the $n^{\prime \prime}$ value except a longer feedback length, since the gain is increased for both cases. Thus, a similar tendency is observed here as that manifested in Fig. 9.

In general, the optimal output should be obtained at a relatively thin emissive CLC cell $(<8 \mu \mathrm{m})$ and the cell gap of CLC reflector used for the external resonator should not be too thick $(<5 \mu \mathrm{m})$ in order to allow $\sim 3-4 \%$ transmittance. Our investigation on the system parameters aims to provide some guidelines for designing the photonic band edge CLC lasers with an external resonator supported. These critical values of the key parameters are based on the current materials and concentrations that we employed. Although the simulations of the $n^{\prime \prime}$ and $d_{1}$ dependencies are only based on three CLC layers without glass substrates, the physical mechanism and the tendency discussed here still hold for the real CLC cells without losing generality.

Our current device configuration of symmetric CLC Fabry-Perot external cavity can be easily extended to asymmetric case where the two CLC reflectors having different cell gaps to achieve a different reflectivity. Laser emission will be accordingly redirected to mainly emit from a single direction rather than both, which will be more attractive for practical applications.
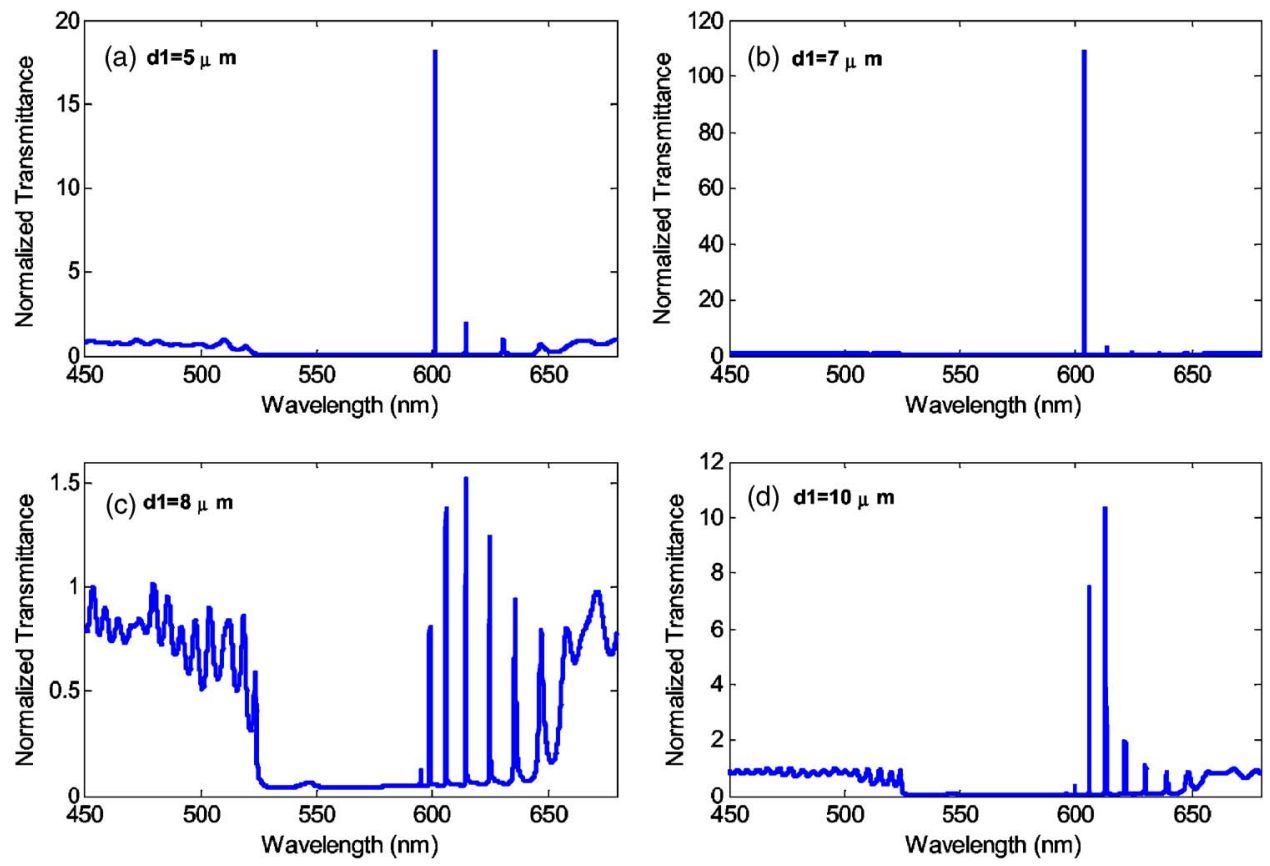

FIG. 10. (Color online) Thickness of the active CLC middle cell $d_{1}$ dependent light amplification performance: (a) $d_{1}=5 \mu \mathrm{m}$; (b) $d_{1}=7 \mu \mathrm{m} ;$ (c) $d_{1}=8 \mu \mathrm{m}$; (d) $d_{1}$ $=10 \mu \mathrm{m}$. The incidence is RCP. 


\section{CONCLUSIONS}

We have demonstrated a CLC photonic band edge laser sandwiched between a circularly polarized Fabry-Perot etalon with dramatically enhanced output. Theoretical analysis confirms that the band edge mode of the middle CLC is effectively supported and enhanced by the external Fabry-
Perot if this mode is within the reflection band of the external resonator. At $1.5 \mathrm{wt}$ \% dye concentration, the optimized output is achieved at a relatively thin active CLC layer and a moderate reflectivity of the resonator reflectors. These analyses are useful in guiding the design of all-organic tunable CLC laser devices with high efficiency and small beam divergence.
[1] V. I. Kopp, Z. Q. Zhang, and A. Z. Genack, Quantum Electron. 27, 369 (2003).

[2] S. T. Wu and D. K. Yang, Reflective Liquid Crystal Displays (Wiley, New York, 2001).

[3] P. Kipfer, R. Klappert, H. P. Herzig, J. Grupp, and R. Dandliker, Opt. Eng. 41, 638 (2002).

[4] J. C. Lee and S. D. Jacobs, J. Appl. Phys. 68, 6523 (1990).

[5] Y. Tanaka, H. Takano, and T. Kurokawa, Jpn. J. Appl. Phys., Part 1 43, 1062 (2004).

[6] Y. Zhou, Y. Huang, A. Rapaport, M. Bass, and S. T. Wu, Appl. Phys. Lett. 87, 231107 (2005).

[7] V. I. Kopp, B. Fan, H. K. M. Vithana, and A. Z. Genack, Opt. Lett. 23, 1707 (1998).

[8] S. Furumi, S. Yokoyama, A. Otomo, and S. Mashiko, Appl. Phys. Lett. 82, 16 (2003).

[9] Y. C. Yang, C. S. Kee, J. E. Kim, H. Y. Park, J. C. Lee, and Y. J. Chon, Phys. Rev. E 60, 6852 (1999).

[10] M. H. Song, K. C. Shin, B. Park, Y. Takanishi, K. Ishikawa, J. Watanabe, S. Nishimura, T. Toyooka, Z. Zhu, T. M. Swager, and H. Takezoe, Sci. Technol. Adv. Mater. 5, 437 (2004).

[11] M. H. Song, B. Park, Y. Takanishi, K. Ishikawa, S. Nishimura, T. Toyooka, and H. Takezoe, Jpn. J. Appl. Phys., Part 1 44,
8165 (2005).

[12] K. Amemiya, M. H. Song, Y. Takanishi, K. Ishikawa, S. Nishimura, T. Toyooka, and H. Takezoe, Jpn. J. Appl. Phys., Part 1 44, 7966 (2005).

[13] Y. Zhou, Y. Huang, and S. T. Wu, Opt. Express 14, 3906 (2006).

[14] R. Ozaki, T. Sanda, H. Yoshida, Y. Matsuhisa, M. Ozaki, and K. Yoshino, Jpn. J. Appl. Phys., Part 1 45, 493 (2006).

[15] Z. Ge, T. X. Wu, X. Zhu, and S. T. Wu, J. Opt. Soc. Am. A 22, 966 (2005).

[16] Y. Huang, T. X. Wu, and S. T. Wu, J. Appl. Phys. 93, 2490 (2003).

[17] E. Hecht, Optics, 2nd edition (Addison-Wesley, Massachusetts, 1987).

[18] A. D’Orazio, V. D. Palo, M. D. Sario, V. Petruzzelli, and F. Prudenzano, Electromagn. Waves 39, 299 (2003).

[19] O. N. Kozina and L. A. Melnikov, Proceedings of the Third Moscow International Symposium on Magnetism, 45 (2005).

[20] K. Ohtaka, J. Lightwave Technol. 17, 2161 (1999).

[21] Y. Huang, T. H. Lin, Y. Zhou, and S. T. Wu, Opt. Express 14, 11299 (2006). 